Itinéraires Itinéraires

Littérature, textes, cultures

2013-1 | 2013

La fiction aujourd'hui

\title{
La règle et le jeu
}

La nouvelle contemporaine comme lieu de réflexion sur la fiction

\section{Claire Colin}

\section{(2) OpenEdition}

\section{Journals}

Édition électronique

URL : http://journals.openedition.org/itineraires/795

DOI : 10.4000/itineraires.795

ISSN : 2427-920X

Éditeur

Pléiade

\section{Édition imprimée}

Date de publication : 1 octobre 2013

Pagination : $51-63$

ISBN : 978-2-343-01791-4

ISSN : $2100-1340$

\section{Référence électronique}

Claire Colin, «La règle et le jeu », Itinéraires [En ligne], 2013-1 | 2013, mis en ligne le 01 octobre 2013, consulté le 19 avril 2019. URL : http://journals.openedition.org/itineraires/795; DOI : 10.4000/ itineraires.795

\section{(c) (i) (9)}

Itinéraires est mis à disposition selon les termes de la licence Creative Commons Attribution - Pas d'Utilisation Commerciale - Pas de Modification 4.0 International. 


\section{La règle et le jeu \\ La nouvelle contemporaine comme lieu de réflexion sur la fiction}

\section{Résumé}

Les stratégies narratives de la nouvelle contemporaine américaine, française et italienne offrent un lieu de réflexion privilégié sur la fiction d'aujourd'hui. Tantôt les nouvellistes exhibent avec ostentation les règles de construction de l'intrigue; tantôt au contraire ils en montrent avec insistance les défauts. S'il s'agit d'introduire de cette façon une distance réflexive sur la fiction, ces procédés ne visent pas à instaurer un nouveau procès du récit mais à faire de la fiction brève un véritable « terrain de jeux narratifs » pour les auteurs.

Mots clés : nouvelle, règles, excès, défauts, jeu

\section{Abstract}

The narrative strategies of contemporary American, French and Italian short stories offer a privileged place of reflection about nowadays fiction. Sometimes short-story writers flaunt the rules of construction of the plot with ostentation; sometimes, on the contrary, they show insistently their flaws. If the intention is to insert in this way a reflexive distance on the fiction, these processes do not aim to institute a new trial of the plot but to make the short fiction a real "field of narrative games" for the authors.

Keywords: short story, rules, excess, flaws, game

À partir des années 1980 on assiste, sinon à une renaissance complète du genre de la nouvelle, tout du moins à un regain d'intérêt des auteurs, lecteurs et éditeurs vis-à-vis du récit bref. Reléguée à l'ombre du roman expérimental ou de l'essai durant les trois précédentes décennies, la nouvelle acquiert à cette date une visibilité renforcée dans le paysage littéraire, même si elle n'atteint pas encore les splendeurs du tournant entre XIX ${ }^{\mathrm{e}}$ et $\mathrm{XX}^{\mathrm{e}}$ siècle (où le genre atteint son apogée, pour reprendre une expression 
de Florence Goyet ${ }^{1}$ ). Lorsqu'Antonio Tabucchi évoque, dans ses entretiens avec Carlos Gumpert, la parution en 1981 de son premier recueil de nouvelles Le Jeu de l'envers, qui lui apporta le succès après la parution de deux romans, il souligne combien le début des années 1980 marque le retour du goût pour la nouvelle, tant chez les auteurs que chez les lecteurs ${ }^{2}$. De même, jetant un regard sur les dix dernières années venant de s'écouler, le géant de la nouvelle américaine Raymond Carver écrit en 1987 :

Les nouvellistes ont-ils jamais connu d'aussi beaux jours? Je ne crois pas. Ou, en tout cas, pas à ma connaissance. Voilà encore dix ans, un auteur débutant qui essayait de placer son premier recueil de nouvelles se trouvait en butte à de terribles difficultés. [...] Aujourd'hui la situation a diamétralement changé, tout le monde le sait bien. [...] La nouvelle est florissante ${ }^{3}$.

Enfin, bien que la situation de la nouvelle en France soit peut-être moins prospère qu'ailleurs, les éditeurs ayant toujours des réticences vis-à-vis du récit bref, à leurs yeux moins rentable financièrement ${ }^{4}$, il n'en demeure pas moins que celui-ci connaît dès le début des années 1980 un renouvellement. Ce dernier se traduit entre autres, comme le notent Annie Mignard et Sabrinelle Bedrane, par un renforcement du genre et une inventivité certaine des formes ${ }^{5}$.

La visibilité retrouvée de la nouvelle n'est pas isolée dans le paysage littéraire. Elle accompagnerait le mouvement plus général constaté à partir de 1980, dont les principales caractéristiques pourraient être l'amoindrissement de l' « aventure d'une écriture », pour reprendre la célèbre expression de Jean Ricardou; non pas la transitivité retrouvée du récit (car au fond, même les œuvres du Nouveau Roman racontent quelque chose), mais la réhabilitation et la force des éléments qui constituent traditionnellement la fiction, comme le personnage, l'intrigue, le réalisme; enfin la capacité des fictions, autrefois remise en question, à appréhender le réel. La publication en 1983 de Temps et récit ${ }^{6}$ par Paul Ricœur est sans doute l'un des indices les plus significatifs d'une telle confiance accordée à nouveau à la fiction organisée en intrigue, encore capable, malgré toutes les transformations

1. Florence Goyet, La Nouvelle, 1870-1925, description d'un genre à son apogée, Paris, PUF, 1993.

2. Antonio Tabucchi et Carlos Gumpert, L'Atelier de l'écrivain, conversations avec Antonio Tabucchi, Genouilleux, La passe du vent, 2001, p. 189.

3. "Sur la littérature actuelle», dans Raymond Carver, N'en faites pas une histoire, Paris, Éditions de l'Olivier, 1994, p. 179-180 (l'article a été publié à l'origine dans la Michigan Quarterly Review, vol. XXVI, n 4, automne 1987).

4. Voir Daniel Grojnowski, Lire la nouvelle, Paris, Nathan, 2000, p. XI.

5. Voir Annie Mignard, La Nouvelle française, Paris, Adpf, 2000, p. 20; Sabrinelle Bedrane, Le Recueil de récits brefs au tournant du second millénaire (1980-2005), hybridité, multiplicité, généricité, thèse de doctorat nouveau régime, littérature française, université Sorbonne nouvelle - Paris 3, 2006, p. 15.

6. Paul Ricœur, Temps et récit, vol. I, L’Intrigue et le récit historique, Paris, Le Seuil, 1983. 
qu'elle a pu subir tout au long du $\mathrm{Xx}^{\mathrm{e}}$ siècle, de répondre à la demande de concordance de la part des lecteurs ${ }^{7}$, se constituant pour l'homme comme le « gardien du temps ${ }^{8} »$.

Toutefois, la nouvelle contemporaine, si elle donne à la fiction une place à nouveau privilégiée, après des années davantage consacrées à la restitution d'un état d'esprit, d'une émotion (pour la «nouvelle-instant») ou bien au travail de l'écriture (pour la « nouvelle-nouvelle $\left.{ }^{9} »\right)$, n'en insère pas moins une distance réflexive vis-à-vis de cette fiction et de ses constituants. Elle se montre ainsi l'héritière des narrations métatextuelles mais en y adoptant une attitude différente vis-à-vis de l'imaginaire fictif mis en place. Les stratégies narratives autour de la notion de contrôle, qu'il s'agisse d'exhiber avec ostentation dans le texte des règles d'écriture, comme pour souligner l'artifice de la composition, ou bien au contraire de souligner dans la narration la perte de maîtrise, peuvent apparaître comme des exemples pertinents de la réflexion à l'œuvre dans la nouvelle. C'est alors non pas tant une confiance envers la fiction qui se manifeste à travers le récit bref contemporain qu'une conscience assumée de ses enjeux.

\section{Jouer des règles excessives}

La nouvelle est un genre particulièrement soumis aux règles, selon la tradition poétique et critique. Nombreux sont les essais consacrés à déterminer les éléments indispensables pour parvenir à un récit bref idéal - un paradoxe lorsque l'on connaît combien il est difficile pour tout théoricien de définir avec précision ce qu'est une nouvelle, ce dont Chklovski fait l'aveu dès le début de son essai consacré au récit bref et au roman ${ }^{10}$. L'article d'Edgar Allan Poe consacré aux Twice Told Tales de Nathaniel Hawthorne ${ }^{11}$, l'un des textes fondateurs de la poétique du genre, insiste par exemple sur la construction nécessairement élaborée à l'extrême de la nouvelle. Celle-ci doit dès la première phrase préparer l'effet final des derniers mots, de façon à proposer un tableau capable de produire, pour celui qui le contemple, " une impression de satisfaction la plus totale ${ }^{12}$ ».

7. «Peut-être faut-il, malgré tout, faire confiance à la demande de concordance qui structure aujourd'hui encore l'attente des lecteurs et croire que de nouvelles formes narratives, que nous ne savons pas encore nommer, sont déjà en train de naître, qui attesteront que la fonction narrative peut se métamorphoser, mais non pas mourir? » (Paul Ricœur, Temps et récit, vol. II, La Configuration dans le récit de fiction [1984], Paris, Le Seuil, 2005, p. 58.) 8. Paul Ricœur, Temps et récit, vol. III, Le Temps raconté [1985], Paris, Le Seuil, 2005, p. 435. 9. Ces termes de «nouvelle-instant» et «nouvelle-nouvelle» sont empruntés à René Godenne (voir René Godenne, La Nouvelle, Paris, Champion, 1995, p. 110-112).

10. Victor Chklovski, « La construction du récit bref et du roman », dans Tzvetan Todorov (dir.), Théorie de la littérature, Paris, Le Seuil, 1966, p. 170.

11. Edgar Allan Poe, « Hawthorne's Twice-Told Tales », Graham's Magazine, 1842.

12. «L'art du conte, Nathaniel Hawthorne », dans Edgar Allan Poe, Contes, essais, poèmes, Paris, Robert Laffont, 1989, p. 1003. 
L'on pourrait s'attendre à ce que la nouvelle contemporaine, après des décennies d'avant-garde bouleversant les traditions littéraires, fasse de la contestation de ces règles d'écriture, ou tout du moins de leur ignorance délibérée, un point de départ. Si certains nouvellistes, en particulier ceux de la « nouvelle-instant », toujours présente même après 1980, adoptent en effet cette démarche, d'autres, au contraire, utilisent une stratégie narrative différente. Les canons d'écriture non seulement demeurent, mais ils sont même exhibés dans l'intrigue avec ostentation. Or, comme le souligne Philippe Daros, en se référant à Jacques Rancière, qu'un régime fictionnel ne soit plus soumis au régime représentatif des arts réglés ne saurait véritablement retenir aujourd'hui l'intérêt, mais qu'un récit mime, ostensiblement, ce régime pour le surexposer pose question ${ }^{13}$.

Ainsi, «L'orecchio assoluto » de Daniele Del Giudice, nouvelle liminaire du recueil Mania ${ }^{14}$, fonctionne sur une série de hasards (hasards de rencontres, hasards de circonstances) qui sont trop récurrents pour ne pas éveiller le soupçon. La fin du récit voit par exemple le narrateur, qui vient de commettre un meurtre, prendre un train pour revenir chez lui et rencontrer le même voyageur croisé à l'aller, lisant et commentant justement un article sur l'assassinat commis la veille... Philippe Daros dans son analyse du récit note :

Le mode d'organisation du muthos, la conduite du récit lui-même se trouvent déterminés par une surexposition paradoxale (explicite ou implicite) d'un principe de causalité d'une totale invraisemblance. L'accomplissement de l'acte de donner la mort, dans les circonstances où il est relaté, s'effectue selon un jeu manifeste de coïncidences dont l'artifice, lui-même tout aussi manifeste, ne peut être réduit par aucune indexation à un quelconque vraisemblable générique [...]. En fait, dans ce récit, le principe de causalité fait l'objet d'un traitement en termes de sous-évaluation (l'inexplicable «psychologique » de la mania) ou de surévaluation, explicitement donnée pour arbitraire (dans la stratégie énonciative) qui en provoque, par excès, la disqualification ${ }^{15}$.

La nouvelle semble désigner ironiquement ce qui la constitue et, de ce fait, en souligner l'artificialité. Il peut être possible de lire la même ironie dans «Fever », de Raymond Carver, publiée dans le recueil Cathedral ${ }^{16}$. Chaque nouvel événement dans la nouvelle est anticipé par un coup de fil d'Eileen à son ex-mari Carlyle : bien que partie du domicile conjugal

13. Philippe Daros, Fictions de reconnaissance, l'art de raconter après la fin des « mythologies de l'écriture »: essai sur l'œuvre de Daniele del Giudice, à paraître aux Éditions de la Galaade.

14. Daniele Del Giudice, Mania, Turin, Einaudi, 1997, p. 5-44.

15. Philippe Daros, op. cit.

16. Raymond Carver, Cathedral [1984], New York, Vintage contemporaries, 1989 et pour la traduction française Les Vitamines du bonheur, trad. Simone Hilling, Paris, Éditions de l'Olivier, 2010. 
pour développer ses projets avec un autre homme, cette femme ressent parfaitement, de loin, les émotions de son mari. Ces intuitions lui permettent de ce fait de prodiguer des conseils et d'anticiper les faits à venir, tant la venue de Mrs Webster, la femme qui saura s'occuper des enfants d'Eileen et Carlyle, que la fièvre qui frappera Carlyle. Un tel sentimentalisme est habituellement absent des nouvelles de Carver, plus connu pour son « sens de la menace » évoqué par maints critiques, et pour l'absence de communication régnant au sein des couples qu'il décrit. Cette première exhibition des rouages de la fiction est en outre renforcée dans le récit par l'extrait suivant, évoquant les cours d'histoire de l'art que donne Carlyle dans son établissement : «At school, they were just leaving the medieval period and about to enter the Gothic. The Renaissance was still some time off, at least not until after the Christmas recess ${ }^{17}$. $\gg$ Laetitia Naly ${ }^{18}$ remarque combien le rythme scolaire de Carlyle se superpose à sa propre vie privée : la sortie du gothique correspond justement à la présence de Mrs Webster, capable de s'occuper des enfants et donc de soulager Carlyle dans sa vie quotidienne. Il n'est pourtant pas au bout de ses peines à cet instant du récit : « la renaissance » définitive interviendra après l'épisode de forte fièvre que connaît le personnage, le poussant à raconter en détail à la nounou toutes les circonstances de sa vie amoureuse passée avec Eileen. Par ce procédé, la nouvelle semble ironiquement désigner sa propre artificialité et, par là, remettre en question l'idée d'un positif possible dans la vie des personnages de Carver, puisque l'univers ici mis en place est ostensiblement fictif, tout du moins trop prévisible.

«La ronde » de Jean-Marie Gustave Le Clézio, nouvelle liminaire du recueil La Ronde et autres faits divers ${ }^{19}$, accentue encore cette exhibition quelque peu ironique des règles d'écriture. Cette nouvelle a souvent fait l'objet de commentaires critiques ${ }^{20}$, notamment pour son possible rapprochement avec la structure du fait divers telle que Roland Barthes l'a décrite $^{21}$. La forte prégnance de l'enchaînement logique et, simultanément,

17. «Fever», dans Raymond Carver, Cathedral, op. cit., p. 176 : «Dans ses cours, il terminait l'époque médiévale et s'apprêtait à aborder le gothique. La Renaissance était encore assez loin, après les vacances de Noël, au plus tôt. »

18. Laetitia Naly, L'Écriture du temps chez Raymond Carver, thèse de doctorat nouveau régime, littérature américaine, université Sorbonne nouvelle - Paris 3, 2008, p. 142.

19. Jean-Marie Gustave Le Clézio, La Ronde et autres faits divers [1981], Paris, Gallimard, coll. « Folio », 2006.

20. On peut ainsi citer l'étude de Bruno Thibault («Du stéréotype au mythe : l'écriture du fait divers dans les nouvelles de J.M.G. Le Clézio », The French Review, vol. 68, n 6 , mai 1995, p. 964-975) tout comme celle d'Isa Van Acker (« Poétique du fait divers, J.M.G. Le Clézio, La Ronde et autres faits divers », dans Paul Pelckmans et Bruno Tristmans (dir.), Écrire l'insignifiant, dix études sur le fait divers dans le roman contemporain, AmsterdamAtlanta, Rodopi, 2000, p. 77-88).

21. «Structure du fait divers» (1962). Cet article sera repris par la suite dans ses Essais critiques, Paris, Le Seuil, 1964, p. 188-197. 
l'événement final inattendu, susceptibles de désigner une sorte de fatum s'acharnant sur les hommes - sans que pour autant ce texte propose une morale explicite à la fin - ont en effet inspiré ce rapprochement. Mais le récit paraît fonctionner aussi comme un pastiche ironique du canon de la nouvelle. Le texte ne cesse de faire allusion à un mécanisme «réglé d'avance ${ }^{22}$ », auquel Martine ne pourra échapper, ce que vient en outre souligner la référence fréquente à des spectateurs anonymes ${ }^{23}$, possible métaphore des lecteurs du récit attendant la fin d'une histoire déjà écrite. Le titre même de la nouvelle, «La ronde », s'il peut faire référence au jeu enfantin qui grise Martine et son amie Titi lorsque, à califourchon sur leurs vélomoteurs lancés à pleine vitesse, elles tracent une ronde autour de la ville, rappelle aussi un système entièrement fermé, dont on ne peut s'échapper. La mise en place d'un engrenage implacable, qui renvoie bien évidemment à la tragédie, n'est pas non plus sans rappeler les préceptes de Poe en ce qui concerne le récit bref, à savoir justement un récit entièrement déterminé dès le début :

Un artiste habile construit un conte. [...] Si sa toute première phrase ne tend pas à amener cet effet, c'est qu'alors dès le tout premier pas, il a fait un faux pas. Dans toute l'œuvre, il ne devrait pas y avoir de mot dont la tendance, de façon directe ou indirecte, ne soit étrangère au dessein préétabli ${ }^{24}$.

Par ailleurs, écrivains et critiques utilisent les comparaisons qui assimilent la nouvelle à un objet de forme circulaire, parce que renvoyant justement à une forme parfaite et refermée sur elle-même ${ }^{25}$. La dernière page de la nouvelle semble exacerber au maximum cette construction entièrement contrôlée du récit. En effet, d'une part le narrateur y décrit les «méandres de sang noir ${ }^{26} \gg$ qui s'échappent des jambes broyées de Martine, fauchée

22. «Puis, tout à coup, à nouveau, la peur revient à l'intérieur de Martine, et sa gorge devient sèche. Elle vient de s'apercevoir que la rue n'est pas vraiment vide, que tout cela est comme réglé d'avance, et qu'elles s'approchent de ce qui va arriver sans pouvoir se détourner » (J.-M. G. Le Clézio, op. cit., p. 17).

23. «Ce qui est terrible surtout, c'est que les gens attendent. Martine ne sait pas où ils sont, ni qui ils sont, mais elle sait qu'ils sont là, partout, le long de la rue, et leurs yeux impitoyables suivent la cavalcade des deux vélomoteurs le long du trottoir » (ibid., p. 16). «Elle voit encore, du coin de l'œil, les visages de ceux qui attendent, qui regardent, les sales embusqués derrière leurs rideaux, derrière leurs autos" (ibid., p. 17). «Il y a les hommes dans la rue, embusqués dans leurs autos arrêtées, cachés derrière les rideaux de leurs chambres » (ibid., p. 23).

24. Edgar Allan Poe, op. cit., p. 1002-1003.

25. Voir les considérations de Julio Cortazar sur le «conte» (qui renvoie plus généralement au «récit bref ») : «Le symbole, la métaphore du conte parfait est la sphère, cette forme sans aucun superflu, qui se ferme totalement sur elle-même [...]. J'ai toujours considéré la nouvelle comme un contenant inexistant, car avant qu'elle soit écrite il n'y a aucun contenant. Mais je sais que lorsque je la termine, son point final doit venir clore cette idée de sphère » (Julio Cortazar, Entretiens avec Omar Prego, Paris, Gallimard, 1984, p. 81).

26. Jean-Marie Gustave Le Clézio, op. cit., p. 24. 
par un camion alors qu'elle vient de commettre un vol à l'arraché. Or, un " méandre » désigne stricto sensu les sinuosités d'un fleuve, ses courbes : l'image du cercle, de la ronde dont on ne peut s'échapper, est ainsi susceptible d'être retrouvée jusque dans le sang de la jeune protagoniste, comme si son sort était, depuis sa naissance, décidé. De la même façon, le texte se termine sur la description du « fermoir de métal doré » du sac à main volé, autre effet d'insistance : le fermoir vient fermer, justement, la nouvelle, comme pour boucler à double tour l'effet de chute. L'insistance de Le Clézio à travers ces images est telle qu'il est possible d'y lire un effet d'ironie vis-àvis des canons de la nouvelle classique, comme un genre dont l'élaboration est entièrement soumise aux règles de construction. " Moloch», le texte qui suit «La ronde », pourrait confirmer cette hypothèse, puisque l'effet de construction ostentatoire y est beaucoup moins important. Le récit, bien plus long, montre une perte progressive des repères spatiotemporels à travers l'histoire de Liana, mettant au monde son enfant dans un mobile home surchauffé, isolée de tous et de tout, avec pour seule compagnie son chienloup Nick. Ce délitement au centre de l'histoire peut souligner justement la capacité de l'écrivain de ne pas faire de l'organisation portée à son point le plus extrême l'élément premier dans la composition de ses nouvelles ${ }^{27}$.

Ce dernier type de jeu peut également s'observer dans le récit bref à travers la présence, directe ou indirecte, de la figure de l'auteur, vue comme une instance capable de diriger à son gré les personnages. « Ragazza Giapponese », l'une des nouvelles du recueil Narratori delle Pianure ${ }^{28} \mathrm{de}$ Gianni Celati, met en scène une jeune femme japonaise qui soumet toutes ses actions aux décisions de son conseiller zodiacal :

Giunta negli Stati Uniti quando aveva 15 anni, s'era sposata quasi subito con un tale di New York e aveva imparato a fare la sarta. S'era presto separata da quel tale, e aveva cominciato a consultare ogni settimana un signist, o consigliere zodiacale, per sapere cosa doveva fare nella vita.

Il consigliere zodiacale le aveva suggerito che, data la posizione di certi astri, l'est non era per lei confacente e sarebbe stato meglio per lei abitare all'ovest. Perciò la ragazza s'era trasferita da New York a Los Angeles [...].

Continuava a consultare ogni settimana per telefono il suo consigliere zodiacale di New York, il quale un giorno le ha detto che per lei sarebbe stato più confacente vivere in una zona collinare. Così la ragazza s'era trasferita al limite nord della città ${ }^{29}[\ldots]$.

27. Pour plus de précisions sur cette présence d'un contrôle exacerbé dans la nouvelle «La ronde », nous nous permettons de renvoyer à notre article «La ronde di Le Clézio: il racconto contemporaneo come doppia possibilità di riflessione », Contemporanea, $\mathrm{n}^{\circ} 9$, 2011, p. 25-34.

28. Gianni Celati, Narratori delle pianure [1985], Milan, Feltrinelli, coll. « Universale Economica », 2008. Pour la traduction française : Narrateurs des plaines, trad. Alain Sarrabayrouse, Paris, Flammarion, 1991.

29. «Ragazza Giapponese », dans Narratori delle Pianure, op. cit., p. 16-17. («Arrivée aux États-Unis à l'âge de quinze ans, elle avait presque aussitôt épousé un type de New 
Ce « professionnel des signes », qui dirige à son gré tous les déplacements du personnage, et apparaît très fréquemment dans la nouvelle, a une référence plutôt métatextuelle, renvoyant à l'auteur. Le contrôle de ce dernier sur son personnage est de cette façon souligné avec insistance, comme pour rappeler combien tout personnage de fiction, simple créature de papier, évolue uniquement selon les directives de son créateur, qui apparaît dans l'histoire, démiurge tout-puissant à l'origine de la production textuelle.

L'exhibition ostentatoire des moyens de production de la fiction occupe donc une place importante dans la nouvelle contemporaine. Il serait alors possible d'avancer que c'est justement le genre de la nouvelle qui permet un tel jeu : parce qu'il s'agit d'un genre considéré comme particulièrement soumis à des règles très strictes (Gianni Celati lui-même souligne cet aspect lorsqu'il évoque le récit bref ${ }^{30}$ ), les nouvellistes contemporains, au lieu de le rejeter, placent cet aspect au centre de leurs textes pour offrir une réflexion à son sujet. Paradoxalement, la fiction, car elle est tout de même présente, se construit autour de ce qui la met à nu.

\section{Jouer des règles défectueuses}

Cette stratégie narrative trouve son exact contraire dans le champ de la nouvelle contemporaine. Certains auteurs - parfois même ceux qui jouent de l'excès des règles dans certains récits - usent du procédé inverse, avec une mise en scène insistante d'une perte de pouvoir dans l'intrigue. Les « défauts » de la fiction sont de cette façon fortement mis en relief.

Le traitement narratif de la temporalité offre ainsi de nombreuses possibilités de dysfonctionnements, qui semblent enrayer le bon déroulement de l'intrigue. La nouvelle « Pausa », dans le recueil Shorts de Vitaliano Trevi$\operatorname{san}^{31}$, met par exemple en scène un joueur de batterie dans un orchestre jazz. Alors qu'il est en train de réaliser avec son groupe un morceau dont l'exécution touche à la perfection, le batteur décide de faire une pause avant le roulement final pour parvenir à l'excellence musicale :

\footnotetext{
York puis avait appris le métier de couturière. Elle s'était bientôt séparée de lui, et avait commencé à consulter chaque semaine un signist, c'est-à-dire un conseiller zodiacal, pour savoir ce qu'elle devait faire dans la vie. / Le conseiller zodiacal lui avait suggéré que, étant donné la position de certains astres, l'est ne lui convenait pas; mieux valait qu'elle habite à l'ouest. C'est pourquoi la fille avait quitté New York pour Los Angeles [...]. / Elle continuait à consulter chaque semaine par téléphone son conseiller zodiacal de New York, qui lui a dit un jour que le mieux pour elle serait d'aller vivre dans une région de collines. Aussi la fille s'était-elle déplacée vers la limite nord de la ville. »)

30. Voir son interview avec Marianne Schneider, «Riscrivere, riraccontare, tradurre », Riga, $\mathrm{n}^{\circ} 28,2008$, p. 48.

31. Vitaliano Trevisan, Shorts, Turin, Einaudi, 2004. Pour la traduction française : Bic et autres shorts, trad. Jean-Luc Defromont Lagrasse, Verdier, 2008.
} 
Tutti aspettano solo me, il colpo di piatti finale, tutto dipende da me. Ma io non volevo che finisse tutto così, capisce, in un modo così banale, la solita rullata, il solito colpo di piatti finale, no, mi dicevo, non può finire così. Farò la rullata, mi dicevo, e tra la rullata e il colpo di piatti ci metto una pausa che non se l'aspetta nessuno. Mi alzai persino in piedi per fare quella rullata, tutto proteso in avanti, i piedi sulla batteria, i muscoli del corpo tesi spasmodicamente. Li avevo tutti in pugno, tutti : il pubblico, $\mathrm{i}$ miei compagni, la musica. Rullata conclusa. Alzai le braccia per dare il colpo di piatti che tutti aspettavano, le bacchette che vibravano, le mani, le braccia, tutto il corpo e il cervello, tutto che vibrava e mi spingeva a dare quel maledetto colpo di piatti. Aspetta, mi dicevo, la pausa dev'essere più lunga, più lunga... Fu allora, disse l'uomo $[\ldots]$ che, tra me e tutti loro, si spalancò quello spaventoso abisso ${ }^{32} \ldots$

Comme le coup de batterie final qui ne vient jamais, l'histoire reste suspendue à cause de la pause temporelle et ne connaîtra jamais de fin conclusive capable de résoudre l'intrigue. De cette façon, le contenu même de l'histoire vient souligner l'attente déçue à la fois des spectateurs et, d'un point de vue plus métatextuel, du lecteur.

Au contraire, les très brèves nouvelles de Microfictions, le recueil de Régis Jauffret ${ }^{33}$, fonctionnent sur une accélération telle du temps que l'action en est comme bâclée : " le récit se suicide avant même de pouvoir s'épanouir », pour reprendre une expression d'Aron Kibedi-Varga ${ }^{34}$. La parodie du roman policier est l'un des aspects les plus représentatifs de cette temporalité trop rapide pour faire parvenir à maturation l'intrigue : l'enquête tourne très rapidement court, le coupable n'étant jamais trouvé. "Un voyage d'un siècle ou deux » part d'une image saisissante : un cadavre de femme dont tous les orifices sont emplis de bijoux précieux. Mais les inspecteurs s'arrêtent presque immédiatement aux personnes les

32. Ibid., p. 16-17 : «Tout le monde n'attend que moi, le coup de cymbales final, tout dépend de moi. Mais moi je ne voulais pas que tout se termine comme ça, d'une façon aussi banale, le coup de cymbales habituel pour finir, non, je me disais, ça ne peut se terminer comme ça. Je ferai le roulement, je me disais, et puis entre le roulement et le coup de cymbale je mets une pause à laquelle personne ne s'attend. Je me suis même levé pour faire le roulement, complètement penché en avant, debout au-dessus de la batterie, les muscles du corps spasmodiquement tendus. Je les tenais tous dans ma main, tous : le public, mes camarades, la musique. Fin du roulement. J'ai levé les bras pour donner le coup de cymbales que tout le monde attendait, les baguettes qui vibraient, les mains, les bras, tout le corps et le cerveau, tout qui vibrait et me poussait à donner ce maudit coup de cymbales. Attends, je me disais, la pause doit être longue, plus longue... C'est à ce moment-là, dit l'homme [...] que cet abîme effrayant s'est ouvert entre moi et tous les autres...»

33. Régis Jauffret, Microfictions [2007], Paris, Gallimard, coll. «Folio», 2009. Nous choisissons d'intégrer dans notre étude de recueils de nouvelles Microfictions, que son auteur préfère qualifier de "roman ». Le format des récits, extrêmement brefs, comme l'absence véritable de liens entre chacun d'entre eux nous semblent autant de raisons valables pour justifier de notre décision.

34. Aron Kibédi-Varga, « Le récit postmoderne », Littérature, n 77, février 1990, p. 20. 
plus proches dans l'entourage de la victime, le gardien de la maison et la caissière du supermarché où le gardien avait l'habitude de faire ses courses, sans chercher à poursuivre plus loin les investigations. La nouvelle s'achève sur ces phrases :

La confrontation avec le vigile n'a rien donné. Après une enquête de moralité, nous avons pu établir que ce Kurde menait une vie d'ascète et envoyait la moitié de sa paye à son père resté dans son pays d'origine. Par contre, la caissière avait un petit ami qui avait été condamné deux ans plus tôt pour vol de voiture. Après l'avoir mise en examen, le juge d'instruction en a référé au Garde des Sceaux. Il a donné son accord pour qu'elle endosse le meurtre, et que cette affaire se termine par une erreur judiciaire ${ }^{35}$.

C'est la généralisation qui justifie l'aboutissement de l'enquête (parce que le petit ami de la caissière a volé une voiture, elle est coupable), dont l'échec est déjà annoncé par l'erreur judiciaire accordée par le Garde des Sceaux. Ce qui semblait prendre les allures d'une intrigue policière classique, à partir d'un meurtre déroutant (le cadavre aux bijoux), tourne au fiasco assumé avec désinvolture par les enquêteurs. « Dans un cercueil à bascule ${ }^{36}$ " pousse sans doute à l'extrême cette parodie du roman policier, puisque le crime, pourtant bien évoqué au début du texte ( «Un assassinat épouvantable, un couteau planté dans la tête $\left.{ }^{37} »\right)$, finit par perdre tout contour précis : " Je me demande où le crime a été commis. On ne m'a pas dit non plus qui était la victime. Et qui était le meurtrier par la même occasion ${ }^{38} »$, constate le narrateur. Il finit par abattre une femme au hasard pour trouver une victime et arrêter tout aussi au hasard un spectateur du meurtre pour lui en faire endosser la responsabilité, avant de conclure : «L'affaire était dans le sac, mais la prochaine fois j'espère que je ne serai pas obligé de me taper en plus de l'enquête le travail qui incombe traditionnellement aux assassins ${ }^{39}$. »

Le dysfonctionnement de la logique narrative, de la configuration précise des faits par l'intrigue, est également un indice de cette perte de contrôle. Plusieurs nouvelles des Microfictions montrent un lien de cause à effet strict en apparence, mais en réalité disproportionné à outrance ou fondé sur la mauvaise foi ou bien encore un tranquille cynisme : "Des reproches, des insultes parfois, et hier un coup de genoux qui m'a laissé pantois. Vous me reprochez la mort de votre épouse, pourtant ce n'est tout de même pas ma faute si elle était malade, et si bien qu'ignorant, on a cru bon de m'accorder un diplôme de médecine », explique le narrateur de

35. Régis Jauffret, op. cit., p. 928.

36. Ibid., p. 161-162.

37. Ibid., p. 161.

38. Ibid.

39. Ibid., p. 162. 
«Conscience de classe ${ }^{40} »$ pour justifier une erreur médicale. Le protagoniste de « Coup de scalpel» affirme pour sa part : « Je n'ai pas tué mes parents par inadvertance, mais parce que je me sentais humilié de leur devoir la vie ${ }^{41}$. »

L'absence d'enchaînement strict des événements au sein de l'intrigue va dans le même sens. Nombre de nouvelles, dans le recueil Cathedral de Raymond Carver, montrent des épisodes dont on détermine mal s'ils sont agencés par un lien de cause à effet ou bien par une simple juxtaposition. Dans « Feathers ${ }^{42} »$, il est difficile de décider si la situation finale, un couple désormais sans aucune complicité après avoir connu une grande proximité, découle de l'épisode central narré dans la nouvelle, un dîner chez des amis, ou d'une autre raison, insaisissable par le narrateur; dans « Careful $^{43}$ ", qui présente une situation générale et une particulière, sans qu'un réel lien soit établi entre les deux, l'hésitation du lecteur demeure : la première englobet-elle la seconde? Vitaliano Trevisan joue également sur ce registre dans Shorts. La nouvelle « Vegetali ${ }^{44}$ » est agencée en deux séquences : la mort d'un homme par arrêt cardiaque et dont l'autopsie révèle l'état déplorable des artères malgré son mode de vie extrêmement sain; la découverte que le champ où il cultivait ses légumes était un ancien charnier. Mais celles-ci n'ont en fin de compte aucun lien entre elles :

Interrogato in proposito, il professor Roberto $\mathrm{T}$. disse che a suo parere [...], il ritrovamento della fossa comune proprio nel campo dove il maestro elementare Franco B. aveva coltivato per anni i suoi vegetali, non era del modo più assoluto da mettersi in relazione con il disastroso stato delle sue arterie e nulla dunque aveva a che fare con la morte per infarto del suddetto maestro ${ }^{45}$.

L'intrigue, écartée entre ces deux épisodes mis en relief, sans qu'il soit possible pourtant de les agencer l'un avec l'autre, souligne elle-même de cette façon son manque de résolution.

Enfin, la configuration mal maîtrisée des événements peut être aussi soulignée par le jeu des répétitions. Les nouvellistes répètent un épisode, ou des actions similaires, comme si l'intrigue était incapable d'avancer véritablement, ou bien font de la répétition l'enjeu central de l'histoire pour

40. Ibid., p. 127.

41. Ibid., p. 137.

42. Raymond Carver, op. cit., p. 3-26.

43. Ibid., p. 111-125.

44. Vitaliano Trevisan, op. cit., p. 115-117.

45. Ibid., p. 117 : «Interrogé à ce sujet, le professeur Roberto T. [...] dit qu'à son avis la découverte de la fosse commune précisément dans le champ où le maître d'école primaire Franco B. avait cultivé pendant des années ses légumes ne devait absolument pas être mise en relation avec l'état désastreux de ses artères, et qu'elle n'avait donc rien à voir avec la mort par infarctus dudit maître. » 
dire l'impossibilité d'aller de l'avant. Certaines nouvelles de Narratori delle pianure prennent la forme initiale d'une quête, telle que trouver un adulte qui ne soit pas ennuyeux dans « Bambini pendolari che si sono perduti ${ }^{46}$ ", parvenir à montrer ses talents dans un match de football ( « Cosa è successo a tre fratelli calciatori $\left.{ }^{47} »\right)$ ou inventer la machine du mouvement perpétuel (« La macchina del moto perpetuo di seconda specie $\left.{ }^{48} »\right)$. Mais cette quête ne trouve jamais d'aboutissement. L'échec, au contraire, se répète à plusieurs reprises, et semble signifier l'impossibilité de mener à bien le projet du début. «Pianista » de Vitaliano Trevisan ${ }^{49}$ met en scène un pianiste de jazz prometteur mais dont tout le jeu est fondé sur une imitation de Bill Evans : le narrateur le lui signale et lui suggère de trouver plutôt son propre style. Quelque temps plus tard, le pianiste reprend ses concerts, et le narrateur se rend à nouveau au local où il joue, mais pour découvrir que le musicien a désormais un jeu fondé sur celui de Chick Corea... Outre le concept d'imitation, capital pour Trevisan dont nombre de romans fonctionnent comme un pastiche assumé du style et des thèmes de Thomas Bernhard, la nouvelle repose aussi sur une répétition, qui souligne ironiquement l'impossibilité pour le personnage du pianiste d'aller au-delà de l'imitation et, pour l'intrigue, le risque de se répéter indéfiniment.

Par le jeu autour de la perte du contrôle dans la configuration de l'intrigue, la nouvelle désigne ce qui la constitue et, simultanément, les possibles faiblesses dans la mise en place du récit. L'on en vient paradoxalement au même constat que pour le jeu autour des règles montrées avec ostentation : la nouvelle s'élabore à partir d'éléments dont on souligne l'usure, et qui malgré tout restent indispensables pour construire la fiction. De même, il est possible de remarquer que les nouvellistes jouent là aussi du genre même de la nouvelle, pour mettre en place le dysfonctionnement de l'intrigue : le synthétisme propre au genre permet d'accumuler de façon ostentatoire des dysfonctionnements qu'un roman ne pourrait peut-être pas mettre autant en avant, et par là d'offrir une réflexion sur ces défauts volontaires de configuration.

\section{Une conscience des règles pour renouveler le jeu}

Ainsi, l'analyse du jeu autour des règles dans la nouvelle contemporaine montre une ostentation, ou au contraire un dérèglement, et permet d'interroger la question de la confiance retrouvée vis-à-vis du récit. Si l'histoire désigne elle-même ironiquement ce qui la constitue, il est en effet difficile d'y attacher seulement les notions de certitude ou de fiabilité. Pour autant, il ne s'agit pas de décrédibiliser l'intrigue ou le personnage, d'en

46. Gianni Celati, op. cit., p. 21-25.

47. Ibid., p. 26-31.

48. Ibid., p. 118-121.

49. Vitaliano Trevisan, op. cit., p. 87-88. 
dénoncer l'artificialité pour la rejeter, d'en souligner la perte de puissance pour célébrer au contraire l'écriture, et de réitérer le procès du récit. Le geste est, plutôt que critique, ludique : on devine un jeu autour des possibilités qu'offre à chaque fois le récit bref pour se jouer des règles. Le titre Mania de Del Giudice, s'il renvoie à la psychologie des personnages affectés justement d'une manie, peut aussi faire référence à la manie même de l'auteur, celle de multiplier les jeux fictifs autour des règles du récit bref. Les titres Microfictions de Régis Jauffret et Shorts de Vitaliano Trevisan vont dans le même sens : il s'agit de présenter un nombre important de " variations fictives », une quarantaine pour Trevisan, jusqu'à cinq cents pour Jauffret, qui permettent un renouvellement continuel des compositions possibles, en jouant autour des dysfonctionnements narratifs. De la même façon, le titre du premier recueil d'Antonio Tabucchi, Il Gioco del roves$c i o^{50}$ indique lui-même l'un des enjeux principaux du livre : multiplier les possibilités ludiques autour d'un même concept : «L'essermi accorto un giorno, per le imprevedibili circostanze della vita, che una certa cosa che era "cosi”" era invece anche in un altro modo ${ }^{51}$. » Le récit bref permet autant de possibilités qu'il y a de nouvelles dans le recueil.

Peut-être pourrait-on avancer l'hypothèse que, plus qu'une confiance, c'est une conscience des possibilités de la fiction qui est désormais ainsi mise en scène. Celle-ci apparaîtrait comme un terrain de jeux, montrant d'une part que l'héritage des années de la métatextualité demeure de cette façon prégnant, et plus encore qu'il ne s'agit plus de refuser les excès ou les artifices de la fiction, pourtant bien réels, mais au contraire de les utiliser pour bâtir l'intrigue. Le genre de la nouvelle représente particulièrement bien ces enjeux actuels de la fiction : d'une part parce qu'elle permet aux auteurs de tirer parti de ses propriétés (récit synthétique, tradition littéraire des règles de composition...) pour en jouer, d'autre part parce qu'un recueil de nouvelles présente plusieurs fictions, ce qui autorise une multiplicité de terrains de jeux potentiels pour les nouvellistes. Le récit bref apparaît donc non seulement comme un lieu de questionnements sur les procédés narratifs contemporains, mais également comme une réponse possible aux interrogations de la fiction d'aujourd'hui.

Claire Colin

Université Sorbonne nouvelle - Paris 3, Sorbonne Paris Cité, CERC

Università degli Studi di Siena

50. Antonio Tabucchi, Il Gioco del rovescio [1981], Milan, Feltrinelli, coll. « Universale Economica », 2006. Pour la traduction : Le Jeu de l'envers [1993], trad. Lise Chapuis, Paris, Gallimard, coll. « Folio », 2006.

51. Ibid., p. 5 : « le fait de m'être un jour aperçu, à cause des imprévisibles événements qui régissent notre vie, que quelque chose qui était "ainsi" pouvait, cependant, être autrement ». 